\title{
EXTENDING HOMEOMORPHISMS BY MEANS OF COLLARINGS
}

\author{
RAYMOND Y. T. WONG ${ }^{1}$
}

1. Introduction. Let $I^{\infty}$ denote the Hilbert cube $\prod_{i=1}^{\infty} I_{i}$, where $I_{i}=I=[0,1]$ with metric $\rho(x, y)=\sum_{i=1}^{\infty}\left|x_{i}-y_{i}\right| / 2^{i}$. If $A, B$ are subsets of a space $X$ such that $A \subset B, A$ is said to be collared (bi-collared) in $B$ if there is a homeomorphism $h$ carrying $A \times[0,1)(A \times(0,1))$ into an open subset of $B$ containing $A$ such that $h(a, 0)=a\left(h\left(a, \frac{1}{2}\right)=a\right)$ for all $a \in A$. We let $H(X)$ denote the group of all homeomorphisms of $X$ onto itself. It is shown in [2] by Wong that if $K$ is a collared subHilbert cube in $I^{\infty}$, then each homeomorphism $h$ of $K$ onto $K_{0}$ can be extended to an $\tilde{h} \in H\left(I^{\infty}\right)$, where $K_{0}=\left\{x \in I^{\infty}: x_{1}=0\right\}$. This result also follows from a Theorem in [1]. The following question was raised in [2]: If $K$ is a bi-collared sub-Hilbert cube in $I^{\infty}$, is it true that each homeomorphism $h$ of $K$ onto $K_{1 / 2}$ can be extended to an $\tilde{h} \in H\left(I^{\infty}\right)$, where $K_{1 / 2}=\left\{x \in I^{\infty}: x_{1}=\frac{1}{2}\right\}$ ? It is the purpose of this paper to answer this question affirmatively. In fact more general situations are studied. We show that if $K$ is a closed subset of $I^{\infty}$ such that each component of $I^{\infty}-K$ is of type $(Q)$ (see below for definition), then a necessary and sufficient condition that a homeomorphism $h$ of $K$ into $I^{\infty}$ be extended to an $\tilde{h} \in H\left(I^{\infty}\right)$ is that $h$ be extended to a neighborhood of $K$ onto a neighborhood of $h(K)$. Theorem 1 gives a necessary and sufficient condition that a closed subset $K$ of $I^{\infty}$ be mapped onto a point by a mapping of $I^{\infty}$ onto itself supported on a neighborhood of $K$ and is 1-1 outside of $K$.

2. Definition. An open subset $V$ of $I^{\infty}$ is of type $(Q)$ if the boundary of $V, \operatorname{Bd}(V)$, is collared in $\bar{V}$ and is homeomorphic to $I^{\infty}$. A mapping is a continuous function. If $f$ is a mapping from a space $X$ into a space $Y, K$ is an inverse set of $f$ if $K$ contains at least two points and for some $y \in Y, K=f^{-1}(y)$. A mapping $f$ of $X$ into itself is to be supported on $C$ if $f$ is the identity on $X-C$. We let $e$ denote the identity mapping for the corresponding space. A closed subset $K$ of $I^{\infty}$ is of type $(P)$ if for each open set $U$ containing $K$, there is a mapping $f$ of $I^{\infty}$ onto itself such that $f$ is supported on $U, 1-1$ outside of $K$ and maps $K$ onto a

Received by the editors July 17, 1967.

1 This research was supported in part by NSF Grant GP-5860. 
point. A continuum $C$ is unicoherent provided that if $C=H \cup K, H$ and $K$ are subcontinua, then $H \cap K$ is connected. Throughout this paper, we shall denote the set $\left\{x \in I^{\infty}: x_{1}=t\right\}$ by $K_{t}$.

3. The following lemmas will be needed to prove Theorem 1 .

LEMMA 1. $I^{\infty}$ is an unicoherent continuum.

Proof. Well known. (See for example, [3, p. 364].)

LEMMA 2. Let $K$ be abi-collared subcontinuum of $I^{\infty}$ and let $h(K \times(0,1))$ be a bi-collared of $K$ in $I^{\infty}$. Then both $K$ and $h\left(K \times\left(\frac{1}{3}, \frac{2}{3}\right)\right)$ separate $I^{\infty}$ into exactly two components.

Proof. Let $D_{1}=h(K \times(0,1 / 3])$ and $D_{2}=h(K \times[2 / 3,1))$. Let $A$ $=h(K \times[1 / 3,2 / 3])$ and $B=I^{\infty}-\operatorname{Int}(\mathrm{A})$. Clearly $A$ is connected and $A \cap B$ is not. Therefore by Lemma $1, B$ must be disconnected. $B$ cannot contain more than two components since each component of $B$ must contain either $D_{1}$ or $D_{2}$. Hence $B$ has exactly two components. It clearly follows that $I^{\infty}-K$ also has exactly two components.

Lemma 3. Let $V=(t, 1] \times \prod_{i=2}^{\infty} I_{i}$, where $0<t<1$ and $(t, 1] \subset I_{1}$. Then there is a mapping $h$ of $I^{\infty}$ onto itself such that $h$ is the identity on $K_{1}, 1-1$ on $V$ and maps $I^{\infty}-V$ onto $0(=(0,0, \cdots))$.

Proof. Let $Q_{0}=I^{\infty}$. For $n \geqq 1$, let $Q_{n}=\prod_{i=1}^{n}\left[0,1 / 2^{n}\right]_{i} \times \prod_{j=n+1}^{\infty} I_{j}$ where each $\left[0,1 / 2^{n}\right]_{i}=\left[0,1 / 2^{n}\right]$ and for $n \geqq 1$, let $C_{n}=\operatorname{Bd}\left(Q_{n}\right)$. It is evident that (1) $\lim _{n \rightarrow \infty} \operatorname{diam}\left(Q_{n}\right)=0$ and (2) for $n>m \geqq 1$, there is a homeomorphism of $I^{\infty}$ onto itself carrying $C_{m}$ onto $C_{n}$ and is the identity on a neighborhood of $K_{1}$. Now let $\left\{t_{i}\right\}_{i \geq 0}$ be a sequence of reals such that $t<\cdots<t_{2}<t_{1}<t_{0}=1$ and $t_{i} \rightarrow t$. Evidently for each $i \geqq 1$, we can choose inductively a homeomorphism $h_{i}$ of $\left[t_{i}, t_{i-1}\right] \times \prod_{j=2}^{\infty} I_{j}$ onto $Q_{i-1}-\operatorname{Int}\left(Q_{i}\right)$ so that $h_{1}$ is the identity on $K_{1}$ and $h_{i}$ agrees with $h_{i+1}$ on $t_{i} \times \prod_{j=2}^{\infty} I_{j}$. Then define $h$ by taking $h=h_{i}$ on each $\left[t_{i}, t_{i-1}\right]$ $\times \prod_{j=2}^{\infty} I_{j}$ and let $h\left(I^{\infty}-V\right)=0$.

Corollary 1. For each closed subset $K$ of $I^{\infty}$ and each $x \in I^{\infty}-K$, there is a neighborhood $V$ of $x$ such that $\bar{V} \cap K=\varnothing$ and for each $\epsilon>0$, there exists an $h \in H\left(I^{\infty}\right)$ satisfying (1) $h$ is the identity on $V$ and (2) $\operatorname{diam}(h(K))<\epsilon$.

Proof. We may assume $x=0$ since $I^{\infty}$ is homogeneous. Now the corollary clearly follows from the method in proving Lemma 3.

Note. We shall from now on denote such a neighborhood $V$ as $N(x, K)$.

Corollary 2. Let $V_{0}^{\prime}=\left\{x \in I^{\infty}: x_{1} \in(3 / 8,5 / 8)\right\}$. Then there is a 
mapping $f$ of $I^{\infty}$ onto itself such that $f$ is the identity on $K_{1 / 2}, 1-1$ on $V_{0}^{\prime}$ and map $A_{0}^{\prime}$ to $0, A_{1}^{\prime}$ to $1(=(1,1, \cdots))$, where $A_{0}^{\prime}, A_{1}^{\prime}$ are components of $I^{\infty}-V_{0}^{\prime}$ such that $0 \in A_{0}^{\prime}$.

Proof. Clear.

4. Theorem 1. Let $K$ be a closed subset of $I^{\infty}$. The following are equivalent:

(i) There is $N(x, K)$ (see $\S 3$, Corollary 1) such that for each open set $U$ containing $K$, there is an open set $V$ such that $K \subset V \subset U$, and an $h \in H\left(I^{\infty}\right)$ such that $h$ is identity on $K$ and carries $I^{\infty}-V$ into $N(x, K)$.

(ii) $K$ is of type $(P)$.

(iii) For each open set $U$ containing $K$, there is an open set $V$ of type (Q) such that $K \subset V \subset U$.

Proof. (i) $\Rightarrow$ (ii). Let $U$ be an open set containing $K$. Choose a sequence of open neighborhoods $\left\{U_{i}\right\}_{i=1}^{\infty}$ about $K$ with the following properties: (1) Each $U_{i} \subset U \cap\left(I^{\infty}-\bar{N}(x, K)\right)$, (2) $U_{i} \supset \bar{U}_{i+1}$, (3) $K=\bigcap_{i=1}^{\infty} U_{i}$ and (4) for each $i$, there is an $h_{i} \in H\left(I^{\infty}\right)$ carrying $I^{\infty}-U_{i}$ into $N(x, K)$ and is the identity on $K$. We now define a sequence $\left\{f_{i}\right\}_{i=1}^{\infty} \subset H\left(I^{\infty}\right)$ as follows: For $\epsilon>0$, let $g_{1} \in H\left(I^{\infty}\right)$ such that $\left.g_{1}\right|_{N(x, K)}$ $=e$ and $\operatorname{diam}\left(g_{1}(K)\right)<\epsilon$. Let $f_{1}=h_{1}^{-1} g_{1} h_{1}$. Then clearly $\left.f_{1}\right|_{I^{\infty}-U_{1}}=e$ and we can choose $\epsilon$ small enough so that $\operatorname{diam}\left(f_{1}(K)\right)<\frac{1}{2}$. Now suppose $f_{i}$ has been defined. For $\epsilon>0$, let $g_{i+1} \in H\left(I^{\infty}\right)$ such that $\left.g_{i+1}\right|_{N(x, K)}=e$ and $\operatorname{diam}\left(g_{i+1}(K)\right)<\epsilon$. Let $f_{i+1}=f_{i} h_{i+1}^{-1} g_{i+1} h_{i+1} f_{i}^{-1}$. It is routine to verify that $f_{i+1}$ is the identity on $f_{i}\left(I^{\infty}-U_{i+1}\right)$. Since $\epsilon$ is arbitrary, we can choose $\epsilon$ small enough so that $f_{i+1}\left(f_{i}(K)\right)<1 / 2^{i+1}$. Now let $f=\cdots f_{2} f_{1}$. It is evident that $f$ exists and is the desired mapping.

(ii) $\Rightarrow$ (iii). Clear.

(iii) $\Rightarrow$ (i). Let $a \in I^{\infty}-K$. Let $U$ be an open set containing $K$. Let $V$ be an open set of type $(Q)$ such that $K \subset V \subset U$. We may suppose $\operatorname{cl}(N(a, k)) \cap \bar{V}=\varnothing$. Let $B=\operatorname{Bd}(V)$. Let $h$ be a homeomorphism of $B \times[0,1)$ onto an open subset of $\bar{V}$ such that $h(b, 0)=b$ for all $b=B$. Let $B_{1 / 2}=h\left(B \times \frac{1}{2}\right)$. Since $B_{1 / 2}$ is homeomorphic to $I^{\infty}$, there is a homeomorphism $g_{0}$ of $h(B \times(0,1))$ onto $V^{\prime}$ carrying $B_{1 / 2}$ onto $K_{1 / 2}$ where $V^{\prime}=\left\{x \in I^{\infty}: x_{1} \in(1 / 3,2 / 3)\right\}$. Let $V_{0}^{\prime}, A_{0}^{\prime}, A_{1}^{\prime}$ and $f$ be defined as in $\$ 3$, Corollary 2. Let $V_{0}=g_{0}^{-1}\left(V_{0}^{\prime}\right)$. Let $A_{0}, A_{1}$ be components of $I^{\infty}-V_{0}$ such that $a \in A_{0}$. We may assume $g_{0}$ is so chosen that $g_{0}\left(A_{0}\right) \cap h(B \times(0,1))=A_{0}^{\prime} \cap V^{\prime}$. Define a mapping $g$ of $I^{\infty}$ onto itself as follows: $g(x)=f g_{0}(x)$ for $x \in V_{0}$ and let $g\left(A_{0}\right)=0, g\left(A_{1}\right)=1$. Then $g$ has exactly two inverse sets $A_{0}$ and $A_{1}$.

We now proceed to eliminate the inverse set $A_{1}$ from $g$. Let $L^{\prime}$ $=\operatorname{Bd}([0,1 / 8] \times[0,1 / 8]) \times \prod_{i=3}^{\infty} I_{i} \subset I^{\infty}$. Let $C$ be the closure of the 
component of $I^{\infty}-L^{\prime}$ containing 1 . Let $x^{\prime}=(1 / 8,0,0, \cdots) \in I^{\infty}$ and $x=g^{-1}\left(x^{\prime}\right)$. Using homogeneity of $I^{\infty}$ and method of Lemma 3 again, there are a neighborhood $W$ about $x$ and an $\alpha \in H\left(I^{\infty}\right)$ such that (1) $\bar{W} \subset V_{0},\left.(2) \alpha\right|_{w}=e$ and (3) $\alpha\left(A_{0}\right) \subset N(a, K)$. Since $g$ is a local homeomorphism at $x$, there is an open set $W^{\prime}$ about $x^{\prime}$ such that $g^{-1}\left(W^{\prime}\right) \subset W$. Evidently there is a homeomorphism $\beta$ of $I^{\infty}$ onto $C$ satisying (1) $\beta$ is the identity on a neighborhood of 1 and (2) $\beta^{-1}\left(L^{\prime}\right) \subset W^{\prime}$. Define

$$
\begin{aligned}
F(x) & =x & & \text { if } x \in A_{1}, \\
& =g^{-1} \beta g(x) & & \text { if } x \notin A_{1} .
\end{aligned}
$$

Then (1) $F$ is a mapping of $I^{\infty}$ into itself, (2) $A_{0}$ is the only inverse set of $F$, (3) if $L=g^{-1} \beta^{-1}\left(L^{\prime}\right)$, then $L \subset W$ and $F(L)=g^{-1}\left(L^{\prime}\right)$, and (4) $F\left(I^{\infty}\right)=g^{-1}(C)=$ closure of the component of $I^{\infty}-F(L)$ that does not contain $a$.

$F\left(I^{\infty}-A_{1}\right)$ is an open set about the point $F\left(A_{0}\right)$. Let $\gamma \in H\left(I^{\infty}\right)$ such that $\gamma$ is the identity on a neighborhood of $F\left(A_{0}\right)$ and carries $F\left(I^{\infty}\right)$ into $F\left(I^{\infty}-A_{1}\right)$. Now define $G$ as follows:

$$
\begin{aligned}
G(x) & =F^{-1} \gamma F(x) & & \text { if } x \notin A_{0}, \\
& =x & & \text { if } x \in A_{\mathbf{0}} .
\end{aligned}
$$

Then (1) $G$ is a homeomorphism of $I^{\infty}$ in to $I^{\infty}-A_{1},(2) G(L)=B d\left(G\left(I^{\infty}\right)\right)$ and (3) $G$ is the identity on a neighborhood of $A_{0}$. Let $h_{0}=G \alpha G^{-1} \cdot h_{0}$ is a homeomorphism of $G\left(I^{\infty}\right)$ onto itself which is the identity on $\operatorname{Bd}\left(B\left(I^{\infty}\right)\right)$ and maps $A_{0}$ into $N(a, K)$. We can extend $h_{0}$ to an $\tilde{h}_{0} \in H\left(I^{\infty}\right)$ by letting $\tilde{h}_{0}$ to be the identity mapping on $I^{\infty}-G\left(I^{\infty}\right)$. By observing $\tilde{h}_{0}\left(I^{\infty}-V\right) \subset \tilde{h}_{0}\left(A_{0}\right) \subset N(a, K)$, we conclude that the proof is completed.

TheOREM 2. If $V \subset I^{\infty}$ is an open set of type $(Q)$, then $\bar{V}$ is homeomorphic to $I^{\infty}$.

Proof. Let $B, h$ and $B_{1 / 2}$ be defined as in proof (iii) $\Rightarrow(\mathrm{i})$. There is a homeomorphism $f$ of $h(B \times[0,1 / 2])$ onto $V_{1 / 2}$ carrying $B$ onto $K_{0}$, $B_{1 / 2}$ onto $K_{1 / 2}$, where $V_{1 / 2}=\left\{x \in I^{\infty}: x_{1} \in[0,1 / 2]\right\}$. Let $U$ be the component of $I^{\infty}-B_{1 / 2}$ such that $B \cap U=\varnothing . \bar{U}$ clearly satisfies (iii), hence is of type $(P)$. Let $g$ be a mapping of $I^{\infty}$ onto itself such that $g$ is supported on $V, 1-1$ outside of $\bar{U}$ and maps $\bar{U}$ onto a point. Let $g^{\prime}$ be a homeomorphism of $I^{\infty}$ onto itself such that $g^{\prime}$ is the identity on $K_{0}, 1-1$ on $\operatorname{Int}\left(V_{1 / 2}\right)$ and maps $I^{\infty}-\operatorname{Int}\left(V_{1 / 2}\right)$ onto 1 . Define a homeomorphism $F$ of $\bar{V}$ onto $I^{\infty}$ as follows: 


$$
\begin{aligned}
F(x) & =g^{\prime} f g^{-1}(x) & & \text { if } x \neq g(\bar{U}), \\
& =1 & & \text { if } x=g(\bar{U}) .
\end{aligned}
$$

Theorem 3. If $K$ is a bi-collared sub-Hilbert cube in $I^{\infty}$, then each homeomorphism $h$ of $K$ onto $K_{1 / 2}$ can be extended to a $\tilde{h} \in H\left(I^{\infty}\right)$.

Proof. This is a consequence of Theorem 2 .

THEOREM 4. If $K$ is a closed subset of $I^{\infty}$ such that each component of $I^{\infty}-K$ is of type $(Q)$, then a homeomorphism $h$ of $K$ into $I^{\infty}$ can be extended to a $\tilde{h} \in H\left(I^{\infty}\right)$ if and only if $h$ can be extended to an open neighborhood of $K$ onto an open neighborhood of $h(K)$.

Proof. Suppose $h$ is extended to a homeomorphism $h^{\prime}$ from a neighborhood of $K$ onto a neighborhood of $h(K)$. Let $\Phi=\{V: V$ is a component of $\left.I^{\infty}-K\right\}$. For each $V \in \Phi$, let $B_{V}=\operatorname{Bd}(V)$ and let $f_{V}$ be a homeomorphism of $B_{V} \times[0,1)$ onto an open subset of $\bar{V}$ such that $f_{V}(b, 0)=b$ for all $b \in B_{V}$. There is a $t_{V}>0$ such that $f\left(B_{V} \times\left[0,1 / t_{V}\right]\right) \subset$ Domain of $h^{\prime}$. Let $K^{\prime}=K \cup\left(\bigcup_{V \in \Phi} f\left(B_{V} \times\left[0,1 / 2 t_{V}\right]\right)\right)$. It is evident that (1) $K^{\prime}$ is compact, (2) $K^{\prime}$ is connected, (3) each component of $I^{\infty}-K^{\prime}$ has a bi-collared boundary which is homeomorphic to $I^{\infty}$ and (4) for each $V \in \Phi, K^{\prime}-f\left(B_{V} \times 1 / 2 t_{V}\right)$ is connected. Now by (2), (3) and (4), components of $I^{\infty}-K^{\prime}$ are in a natural way one to one corresponding with the components of $I^{\infty}-h^{\prime}\left(K^{\prime}\right)$. By method used in Theorem 2, $h^{\prime}$ can be extended to each component of $I^{\infty}-K^{\prime}$ onto the corresponding component of $I^{\infty}-h^{\prime}\left(K^{\prime}\right)$. This observation suffices to conclude the Theorem.

Question. If $K$ is a bi-collared subcontinuum of $I^{\infty}$ such that the closure of each component of $I^{\infty}-K$ is homeomorphic to $I^{\infty}$, is $K$ necessary homeomorphic to $I^{\infty}$ ?

\section{REFERENCES}

1. R. D. Anderson, On topological infinite deficiency, Michigan Math. J. 14 (1967), 365-383.

2. R. Wong, On homeomorphisms of infinite dimensional product spaces, Dissertation, Louisiana State University, 1966.

3. J. Dugundji, Topology, Allyn and Bacon, Boston, Mass., 1966.

University of California, Los Angeles 